PROCEEDINGS OF THE

AMERICAN MATHEMATICAL SOCIETY

Volume 127, Number 12, Pages 3503-3511

S 0002-9939(99)04968-0

Article electronically published on May 13, 1999

\title{
DERIVATIONS AND THE INTEGRAL CLOSURE OF IDEALS
}

\author{
REINHOLD HÜBL AND APPENDIX BY IRENA SWANSON
}

(Communicated by Wolmer V. Vasconcelos)

\begin{abstract}
Let $(R, \mathfrak{m})$ be a complete local domain containing the rationals. Then there exists an integer $l$ such that for any ideal $I \subseteq R$, if $f \in \mathfrak{m}, f \notin I^{n}$, then there exists a derivation $\delta$ of $R$ with $\delta(f) \notin I^{n+l}$.
\end{abstract}

This note grew out of a question raised by C. Huneke some years ago: Suppose $(R, \mathfrak{m})$ is a complete local domain with perfect residue class field $k$ which it contains. Call an element $x \in R$ derivationally constant for $R / k$ if $\delta(x)=0$ for all $k$-derivations $\delta \in \operatorname{Der}_{k}(R)$, and denote by $C(R / k)$ the subring of derivationally constant elements of $R / k$. In this context Craig Huneke asked:

(1) If $I \subseteq R$ is an ideal, does there exist a constant $l=l(R, I) \in \mathbb{N}$ with the following property: If $x \in R$ with $\delta(x) \in I^{n+l}$ for all $\delta \in \operatorname{Der}_{k}(R)$, then there exists a $c \in C(R / k)$ with $x-c \in I^{n}$ ?

(2) Is it possible to bound $l$ in (1) in some uniform way?

Here (2) is meant to cover the situation arising via the techniques of tight closure and reduction $\bmod p$ : If $I$ arises via reduction $\bmod p$ from an ideal $\mathfrak{I}$ given in an algebra $\mathfrak{R}$ containing the integers, then Huneke asks for a bound $l=l(\mathfrak{I})$ working for the reductions modulo all but finitely many primes $p$.

Originally this question arose in the study of rational and $F$-rational singularities and the relations between them (cf. $[\mathrm{F}]$ ), and interest in it has been revived by recent attempts to prove Kodaira vanishing with tight closure techniques (cf. [HS]). In [FHH] a positive answer to (1) was given in case $\operatorname{char}(k)=0$ and $I \subseteq R$ is an $\mathfrak{m}$-primary and one-fibred ideal. Some partial results in positive characteristics are available, for instance in the graded case $([\mathrm{F}])$ and for regular local rings. In this note we will prove the following generalization of $[\mathrm{FHH}],(1.6)$ :

Theorem. Let $k$ be a field, $\operatorname{char}(k)=0$ and let $(R, \mathfrak{m}) / k$ be a local domain such that the universally finite derivation $\widetilde{d}_{R / k}: R \rightarrow \widetilde{\Omega}_{R / k}^{1}$ exists. Then there exists a constant $l=l(R)$ with the following property: If $I \subseteq R$ is an ideal and $r \in \mathfrak{m}$ is an element with $\delta(r) \in I^{n+l}$ for all $\delta \in \operatorname{Der}_{k}(R)$, then $r \in I^{n}$.

This completely answers (1), and it also gives a uniform bound (as asked for in (2)), depending on the ring only. A first attempt to solve this problem would be

Received by the editors November 20, 1997 and, in revised form, February 24, 1998.

1991 Mathematics Subject Classification. Primary 13N05, 13J10.

Key words and phrases. Kähler differentials, derivations.

The author was partially supported by a Heisenberg-Stipendium of the DFG.

The author of the appendix was partially supported by the National Science Foundation.

(C)1999 American Mathematical Society 
to try to generalize $[\mathrm{FHH}],(1.4)$. This however only gives a weaker result which in addition depends on $I$ (cf. the appendix by I. Swanson).

\section{Derivations AND Differential FORMS}

Let $k$ be a noetherian ring and let $R / k$ be an algebra. Throughout this paper we will assume that the universally finite derivation of $R / k$ exists (cf. [KD], $\S 11$ or [SS1]), i.e. we assume that there exists a finite $R$-module $\widetilde{\Omega}_{R / k}^{1}$ and a $k$-derivation

$$
\widetilde{d}_{R / k}: R \longrightarrow \widetilde{\Omega}_{R / k}^{1}
$$

with the following universal property:

If $M$ is a finite $R$-module and $\delta: R \rightarrow M$ is a $k$-derivation, then there exists a unique $R$-homomorphism $f: \widetilde{\Omega}_{R / k}^{1} \rightarrow M$ with $\delta=f \circ \widetilde{d}_{R / k}$.

1.1 Remark. A universally finite $k$-derivation exists in each of the following cases:

(1) $R / k$ is essentially of finite type. In this case the universal derivation of $R / k$ is universally finite.

(2) $k$ is a field with a valuation and $R$ is an analytic $k$-algebra, i.e. $R$ is finite over some ring $k\left\langle\left\langle X_{1}, \ldots, X_{n}\right\rangle\right\rangle$ of convergent power series over $k$.

(3) $k$ is a field, $R$ is complete and $R / \mathfrak{m}$ is a finitely generated field extension of $k$.

Proof. (1) is obvious and (2) is proved in [BKKN]. As for (3) choose $y_{1}, \ldots, y_{t}$ in $R$ whose residue classes form a transcendence basis of $R / \mathfrak{m}$ over $k$ and a system of parameters $x_{1}, \ldots, x_{d}$ of $R$. Then $R$ is finite over $k\left(y_{1}, \ldots, y_{t}\right)\left[\left[x_{1}, \ldots, x_{d}\right]\right]$ by the Nakayama lemma for complete rings ([KD], (12.8)). The universally finite differential module of $k\left(y_{1}, \ldots, y_{t}\right)\left[\left[x_{1}, \ldots, x_{d}\right]\right]$ exists by [KD], (12.5), and therefore $\widetilde{\Omega}_{R / k}^{1}$ exists by $[\mathrm{KD}],(11.9)$.

1.2 Remark. If $k$ is a field, $\operatorname{char}(k)=0$, the existence of $\widetilde{\Omega}_{R / k}^{1}$ implies that $R$ is excellent (cf. [SS1], Satz (8.10))

Assume now in addition that $R$ is a local domain with field of fractions $L=Q(R)$. As in $[\mathrm{FHH}]$ we denote by $\widetilde{\Omega}_{R / k}^{*}$ the module $\widetilde{\Omega}_{R / k}^{1} /$ tor sion, i.e. the image of $\widetilde{\Omega}_{R / k}^{1}$ by the canonical map

$$
\widetilde{\Omega}_{R / k}^{1} \longrightarrow \widetilde{\Omega}_{R / k}^{1} \otimes_{R} L=D_{k}(L)
$$

with $D_{k}(L)$ as in [SS1], resp. [KD], $\S 13$. By $d^{*}$ we denote the composition

$$
R \stackrel{\widetilde{d}_{R / k}}{\longrightarrow} \widetilde{\Omega}_{R / k}^{1} \stackrel{\operatorname{can}}{\longrightarrow} \widetilde{\Omega}_{R / k}^{*}
$$

1.3 Proposition (Universal property of $\widetilde{\Omega}_{R / k}^{*}$ ). $\widetilde{\Omega}_{R / k}^{*}$ is an $\mathfrak{m}$-adically separated and torsion-free $R$-module, and if $M$ is an $\mathfrak{m}$-adically separated and torsionfree $R$-module, and $\delta: R \rightarrow M$ is a $k$-derivation, then there exists a unique $R$-homomorphism $f: \widetilde{\Omega}_{R / k}^{*} \rightarrow M$ with $\delta=f \circ d^{*}$. Hence we have a canonical isomorphism

$$
\operatorname{Hom}_{R}\left(\widetilde{\Omega}_{R / k}^{*}, M\right) \rightarrow \operatorname{Der}_{k}(R, M), \quad f \longmapsto f \circ d^{*} .
$$

Proof. Clearly for any $f \in \operatorname{Hom}_{R}\left(\widetilde{\Omega}_{R / k}^{*}, M\right)$ we have $f \circ d^{*} \in \operatorname{Der}_{k}(R, M)$. Thus it remains to show the first part of the proposition. By the universal property of the module $\Omega_{R / k}^{1}$ of Kähler differentials, there exists $F_{1}: \Omega_{R / k}^{1} \rightarrow M$ with 
$\delta=F_{1} \circ d_{R / k}$. As $F_{1}$ is continuous for the $\mathfrak{m}$-adic topology and as $M$ is separated, $F_{1}\left(\bigcap_{n \in \mathbb{N}} \mathfrak{m}^{n} \Omega_{R / k}^{1}\right)=0$. Therefore $F_{1}$ induces a map

$$
F_{2}: \widetilde{\Omega}_{R / k}^{1}=\Omega_{R / k}^{1} /\left(\bigcap_{n \in \mathbb{N}} \mathfrak{m}^{n} \Omega_{R / k}^{1}\right) \longrightarrow M
$$

(cf. $[\mathrm{KD}],(11.13))$. Since $M$ is torsion-free, $F_{2}$ induces $f: \widetilde{\Omega}_{R / k}^{*} \rightarrow M$ as desired.

In the proof of the theorem we will also need the following:

1.4 Propositon. Let $k$ be a field, $\operatorname{char}(k)=0$, and let $(R, \mathfrak{m}) / k$ be a complete local domain such that $R / \mathfrak{m}$ is a finitely generated field extension of $k$. Then there exists a constant $l_{1}=l_{1}(R)$ with the following property:

If $I \subseteq R$ is an ideal and $z \in R$ is an element with $d^{*} z \notin I^{n} \widetilde{\Omega}_{R / k}^{*}$, then there exists a $\delta \in \operatorname{Der}_{k}(R)$ with $\delta(z) \notin I^{n+l}$.

Proof (cf. [FHH], (1.7)). Let $y_{1}, \ldots, y_{t}$ be elements in $R$ whose residue classes form a transcendence basis of $R / \mathfrak{m}$ over $k$, let $x_{1}, \ldots, x_{d}$ be a system of parameters of $R$, and set $L=Q(R)$. Then the images of $d^{*} y_{1}, \ldots, d^{*} y_{t}, d^{*} x_{1}, \ldots, d^{*} x_{d}$ form an $L-$ basis of $\widetilde{\Omega}_{R / k}^{*} \otimes_{R} L$ as $\operatorname{char}(k)=0$ and $L$ is finite over $Q\left(k\left(y_{1}, \ldots, y_{t}\right)\left[\left[x_{1}, \ldots, x_{d}\right]\right]\right)$. Hence $d^{*} y_{1}, \ldots, d^{*} y_{t}, d^{*} x_{1}, \ldots, d^{*} x_{d}$ generate a free $R$-submodule $F$ of $\widetilde{\Omega}_{R / k}^{*}$ with $F \otimes_{R} L=D_{k}(L)$. Thus there exists an $r \in R$ with $r \cdot \widetilde{\Omega}_{R / k}^{*} \subseteq F$. By Huneke's uniform Artin-Rees Lemma [Hn], (4.12), there exists an $l_{1}=l_{1}(R)$ such that for any ideal $I \subseteq R$ and any $n \in \mathbb{N}$ :

$$
I^{n+l_{1}} \cdot \widetilde{\Omega}_{R / k}^{*} \cap r \cdot \widetilde{\Omega}_{R / k}^{*} \subseteq I^{n} \cdot r \cdot \widetilde{\Omega}_{R / k}^{*} .
$$

Hence if $z \in R$ with $d^{*} z \notin I^{n} \cdot \widetilde{\Omega}_{R / k}^{*}$, then $r d^{*} z \in F \backslash I^{n+l_{1}} \cdot F$. Thus there exists $l \in \operatorname{Hom}_{R}(F, R)$ with $l\left(r d^{*} z\right) \notin I^{n+l_{1}}$ as $F$ is free, and the composition

$$
\widetilde{\Omega}_{R / k}^{*} \stackrel{\mu_{r}}{\longrightarrow} r \cdot \widetilde{\Omega}_{R / k}^{*} \subseteq F \stackrel{l}{\longrightarrow} R
$$

determines via (1.3) a $\delta \in \operatorname{Der}_{k}(R)$ as desired.

1.5 Remark. The proof of (1.4) actually proves a uniform version of [FHH], (1.7): If $M$ is a finite and torsion-free $R$-module, then there exists an $l \in \mathbb{N}$ with the following property: If $I \subseteq R$ is an ideal and if $m \in M, m \notin I^{n} M$, then there exists a homomorphism $f \in \operatorname{Hom}_{R}(M, R)$ such that $f(m) \notin I^{n+l}$.

1.6 Corollary. In the situation of (1.4) $C(R / k)=\bar{k}$, the algebraic closure of $k$ in (any choice of) a coefficient field of $R / k$. In particular if $k \cong R / \mathfrak{m}$, then $C(R / k)=k$.

Proof. Clearly $\bar{k} \subseteq C(R / k)$, as $\bar{k} / k$ is algebraic and separable. Now suppose that $z \in R$ and either $z \in \mathfrak{m}$ or the residue class of $z$ in $R / \mathfrak{m}$ is transcendental over $k$. Then either $z$ is part of a system of parameters of $R$ or the residue class of $z$ is part of a transcendence basis of $R / \mathfrak{m}$ over $k$. In either case $d^{*} z \neq 0$ in $\widetilde{\Omega}_{R / k}^{*}$ (as in the proof of (1.4)) and the claim follows from (1.4) (applied to a sufficiently high power of $\mathfrak{m})$.

1.7 Remark. In the situation of (1.4) assume that $z \in R$ is an element such that its residue class $\bar{z} \in R / \mathfrak{m}$ is transcendental over $k$. Then there exists a $\delta \in \operatorname{Der}_{k}(R)$ such that $\delta(z) \in R^{*}$ is a unit. 
Proof. As $\operatorname{char}(k)=0$, there exists a coefficient field $K \subseteq R$ containing $z$. Then $\operatorname{Der}_{k}(K) \subseteq \operatorname{Der}_{k}(K, R) \subseteq \operatorname{Der}_{k}(R)$ (the latter as $\widetilde{\Omega}_{R / k}^{*} \stackrel{\text { can }}{\longrightarrow} \Omega_{R / \mathfrak{m} / k}^{1} \cong \Omega_{K / k}^{1}$ is surjective). The assumptions imply that $d_{K / k}(z) \neq 0$, and therefore there exists a $\delta \in \operatorname{Der}_{k}(R)$ with $\delta(z) \in K \backslash\{0\}$.

1.8 Remark. (1.4) holds true if $\operatorname{char}(k)=p>0$ as well or if $R$ is not complete. If in addition $k$ is perfect, then $C(R / k)=L^{p} \cap R$ in this case. If furthermore $R$ is normal, then $C(R / k)=R^{p}$.

\section{Proof of THE THEOREM}

Throughout this section we assume that we are in the situation of the theorem. Let $J \subseteq R$ be an ideal. Recall that $x \in R$ is called integral over $J$ if it satisfies an equation $x^{n}+a_{1} x^{n-1}+\cdots+a_{n}=0$ with $a_{l} \in J^{l}$ for $j=1, \ldots, n$. The elements of $R$ which are integral over $J$ form an ideal $\bar{J}$, called the integral closure of $J$.

The crucial ingredient in the proof of the theorem will be the following strengthening of [SS1], (8.11) (see also [SS2], (5.1)), which relates integral closure to derivations:

2.1 Proposition. Let $\mathfrak{A}$ and $J$ be two proper ideals of $R$ and suppose that

$$
d^{*} \mathfrak{A} \subseteq R d^{*} J+\mathfrak{A} \widetilde{\Omega}_{R / k}^{*} .
$$

Then

$$
\mathfrak{A} \subseteq \bar{J}
$$

Proof. Since $R$ is universally catenary by (1.2), it suffices by [Li], (1.1), to show the following: If $L=Q(R)$ and if $v$ is a prime divisor of $R$ with valuation $\operatorname{ring} V \subseteq L$, i.e. $V$ is a discrete valuation ring with $R \subseteq V$ and $\mathfrak{m}=\mathfrak{m}_{V} \cap R$ (cf. [Li], $\S 1$ ), then $\mathfrak{A} V \subseteq J V$. To do so it obviously suffices to show $\mathfrak{A} \hat{V} \subseteq J \hat{V}$ where $\hat{V}$ denotes the $\mathfrak{m}_{V}$-adic completion of $V$. By Cohen's structure theorem we can write $\hat{V}=K[[X]]$ with a field $K \supseteq k$. Now denote by $\delta$ the $k$-derivation

$$
R \stackrel{\operatorname{can}}{\longrightarrow} \hat{V} \stackrel{\frac{\partial}{\partial X}}{\longrightarrow} \hat{V} .
$$

As $\hat{V}$ is an $\mathfrak{m}$-adically separated and torsion-free $R$-module, there exists an $R$ linear homomorphism $f: \widetilde{\Omega}_{R / k}^{*} \rightarrow \hat{V}$ such that $\delta=f \circ d^{*}$ by (1.3). Hence we get

$$
\delta(\mathfrak{A})=f\left(d^{*} \mathfrak{A}\right) \subseteq f\left(R d^{*} J+\mathfrak{A} \widetilde{\Omega}_{R / k}^{*}\right) \subseteq \delta(J) \hat{V}+\mathfrak{A} \hat{V} .
$$

Since $\mathfrak{A} \hat{V}$ is a proper ideal of $\hat{V}$, we have $\delta(\mathfrak{A}) \hat{V} \not \subset \mathfrak{A} \hat{V}$ (for if $a \in \mathfrak{A}$ with $\hat{v}(a)=$ $\min \{\hat{v}(\tilde{a}): \tilde{a} \in \mathfrak{A}\}$, then $\hat{v}(\delta a)=\hat{v}(a)-1<\min \{\hat{v}(\tilde{a}): \tilde{a} \in \mathfrak{A}\}$ since $\operatorname{char}(k)=0)$. Hence we have to have $\delta(\mathfrak{A}) \hat{V} \subseteq \delta(J) \hat{V}$ implying that $\mathfrak{A} \hat{V} \subseteq J \hat{V}$, as desired.

2.2 Corollary. Let $r \in \mathfrak{m}$ with $d^{*} r \in J \widetilde{\Omega}_{R / k}^{*}$. Then $r \in \bar{J}$.

Proof. Apply (2.1) to $\mathfrak{A}=J+r R$ :

$$
d^{*} \mathfrak{A}=d^{*}(J+r R) \subseteq R d^{*} J+R d^{*} r+r \widetilde{\Omega}_{R / k}^{*} \subseteq R d^{*} J+\mathfrak{A} \widetilde{\Omega}_{R / k}^{*} .
$$

The integral closure $\bar{J}$ of an ideal $J$ is "not very much bigger" than the ideal $J$ itself. More precisely, in our situation the assumptions of [Hn], (4.13), are satisfied, and therefore 
2.3 Remark. There exists a constant $l_{2}=l_{2}(R)$ such that for any ideal $I \subseteq R$ and any $n \in \mathbb{N}$ we have: $\overline{I^{n+l_{2}}} \subseteq I^{n}$.

Proof of the theorem. Let $l_{1}$ be as in (1.4), resp. (1.8), $l_{2}$ as in (2.3), and set $l:=l_{1}+l_{2}$. If $r \in \mathfrak{m}, r \notin I^{n}$, then $d^{*} r \notin I^{n+l_{2}} \cdot \widetilde{\Omega}_{R / k}^{*}$ as otherwise $r \in \overline{I^{n+l_{2}}} \subseteq I^{n}$ by $(2.2) /(2.3)$. Thus there exists a $\delta \in \operatorname{Der}_{k}(R)$ with $\delta(r) \notin I^{n+l_{1}+l_{2}}=I^{n+l}$ by (1.4).

2.4 Remark. i) If $R$ is regular, then $\widetilde{\Omega}_{R / k}^{*}$ is free. Thus we may take $l_{1}=0$ in this case. Furthermore we may take $l_{2}=\operatorname{dim}(R)-1$ by the Briancon-Skoda theorem (cf. [LiS]); hence $l=\operatorname{dim}(R)-1$.

ii) If $R$ is a pseudorational local ring, and if we restrict ourselves to the class of $\mathfrak{m}$-primary ideals, then we may take $l_{2}=\operatorname{dim}(R)-1$ by [LiT].

iii) If $(R, \mathfrak{m})$ is a positively graded regular local ring (in the sense of [SW], 33 ), and if we restrict ourselves to the class of homogeneous ideals, then the theorem holds in this context as well, and we may take $l=0$ as follows from [SW], (5.1).

In the case of a complete local ring the theorem can be improved slightly:

2.5 Theorem. Suppose that in the situation of the theorem $(R, \mathfrak{m})$ is a complete local domain. Then there exists a constant $l=l(R)$ with the following property: If $I \subseteq R$ is an ideal and $z \in R$ is an element with $\delta(z) \in I^{n+l}$ for all $\delta \in \operatorname{Der}_{k}(R)$, then there exists a $c \in C(R / k)$ with $z-c \in I^{n}$.

Proof. If $\delta(z) \in I^{n+l}$ for all $k$-derivations $\delta$, then the residue class $\bar{z} \in R / \mathfrak{m}$ in not a transcendental element over $k$ in view of (1.7). Thus by (1.6) there exists a $c \in C(R / k)$ with $z-c \in \mathfrak{m}$. Now we may apply the theorem.

Some of the techniques applied here carry over to positive characteristics as well. In this case some partial results are available for regular local rings. For many applications of tight closure techniques an affirmative answer to the following question would be sufficient:

2.6 Question. Let $(R, \mathfrak{m})$ be a complete regular local ring with perfect residue class field $k$, which it contains. Let $I \subseteq R$ be an ideal and let $z \in R$ be an element with $\delta(z) \in I$ for all $\delta \in \operatorname{Der}_{k}(R)$. Does there exist a $c \in C(R / k)$ with $z-c \in \bar{I}$ ?

\section{ApPEndix: Zeros of DIFFERENTIALS ALONG IDEALS}

This appendix was motivated by Fedder, Huneke and Hübl's analysis of zeros of differentials along one-fibered ideals, that is, ideals with only one Rees valuation (see $[\mathrm{FHH}]$ ). Both this appendix and R. Hübl's paper remove the one-fibered assumption, but the two approaches are different. The key result here, as in $[\mathrm{FHH}]$, is the existence of certain ideals containing a high power of a given ideal (see Theorem A1), which then implies the existence of derivations with a controlled degree of nilpotency along an ideal (Theorem A2). However, the "control" function in Theorem A2 obtained in this way is linear (of arbitrary slope) and depending on the ideal, whereas R. Hübl's approach bypassing any analog of Theorem A1 produces a linear "control" function of slope one which is independent of the ideal. Examples below show that Theorem A1 cannot be improved to produce a "control" function independent of the ideal. However, Theorem A1 has other corollaries and uses (see below). 
Theorem $\mathbf{A 1}$ (cf. [FHH, Theorem 1.4]). Let $(R, \mathfrak{m})$ be a d-dimensional complete local domain, let $I$ be an $\mathfrak{m}$-primary ideal in $R$ and suppose that $R$ has an infinite residue field. Then there exist positive integers $l$ and $k$ satisfying the following:

If $f \in \mathfrak{m}$ and $f \notin I^{n}$, then there exist $g_{2}, \ldots, g_{d} \in I$ such that $I^{k n+l} \subseteq$ $\left(f, g_{2}, \ldots, g_{d}\right)$.

Proof. When $d=1$, the integral closure of $R$ is local so that by [FHH, Lemma 1.3] and [FHH, Theorem 1.4] there exists an integer $l$, independent of $f$, such that $I^{n+l} \subseteq(f)$.

Now let $d>1$. As in the proof of [FHH, Theorem 1.4] we may replace $I$ by its minimal reduction to be able to assume that $I=\left(x_{1}, \ldots, x_{d}\right)$.

Let $v_{1}, \ldots, v_{t}$ be all the Rees valuations of $I$. By (E), page 409, in [R1], there exists a positive integer $C$ such that for any $i, j \in\{1, \ldots, t\}$ and any nonzero element $x$ of $R, v_{i}(x) \leq C v_{j}(x)$. Under the assumption that $I$ has more than one Rees valuation, $C$ has to be 2 or larger. By [R2] there exists an integer $D$, depending only on $I$, such that for all $n \geq D$, the integral closure $\overline{I^{n}}$ is contained in $I^{n-D}$. By assumption $f \notin I^{n}$. Thus $f \notin \overline{I^{n+D}}$ and so for at least one $i \in\{1, \ldots, t\}$, say $i=1, v_{1}(f)<(n+D) v_{1}(I)$. Hence for all $i \in\{1, \ldots, t\}$,

$$
v_{i}(f) \leq C v_{1}(f) \leq C(n+D) v_{1}(I) \leq C^{2}(n+D) v_{i}(I) .
$$

Let $S=R\left[\frac{x_{2}}{x_{1}}, \ldots, \frac{x_{d}}{x_{1}}\right]$ and let $T$ be the integral closure of $S$. Each valuation $v_{i}$ corresponds uniquely to a prime ideal $\mathfrak{P}_{i}$ in $T$ which is minimal over $I T$. The assumption that $v_{i}(f)<C^{2}(n+D) v_{i}(I)$ implies that $f \notin I^{C^{2}(n+D)} T_{\mathfrak{P}_{i}}$. As $T_{\mathfrak{P}_{i}}$ is a one-dimensional normal analytically unramified domain, by the case $d=1$ in [FHH, Theorem 1.4] there exists an integer $E_{i}$, only depending on $I$, such that $I^{C^{2}(n+D)+E_{i}} T_{\mathfrak{P}_{i}} \subseteq(f) T_{\mathfrak{P}_{i}}$. Let $E=\max \left\{E_{1}, \ldots, E_{t}\right\}$. Then for all $i$, $I^{C^{2}(n+D)+E} T_{\mathfrak{P}_{i}} \subseteq(f) T_{\mathfrak{P}_{i}}$.

Let $\mathfrak{Q}_{1}, \ldots, \mathfrak{Q}_{s}$ be all the prime ideals in $S$ minimal over $I S$. Then by construction, as $S \subseteq T$ is module-finite, for all $i=1, \ldots, s$,

$$
I^{C^{2}(n+D)+E} S_{\mathfrak{Q}_{i}} \subseteq\left(\bigcap_{\mathfrak{P}_{j} \cap S=\mathfrak{Q}_{i}}(f) T_{\mathfrak{P}_{j}}\right) \cap S_{\mathfrak{Q}_{i}} \subseteq \overline{(f) S_{\mathfrak{Q}_{i}}},
$$

the last ideal being the integral closure of $(f) S_{\mathfrak{Q}_{i}}$.

Now we apply Proposition 5.5 from Huneke's [Hn]: for each $i=1, \ldots, s$, there exists an integer $F_{i}$, depending only on $S_{\mathfrak{Q}_{i}}$ and therefore on $I$, such that for all ideals $J$ in $S_{\mathfrak{Q}_{i}}$ and all integers $n$, the integral closure of $J^{n}$ is contained in $J^{n-F_{i}}$. Let $F=\max \left\{F_{1}, \ldots, F_{s}\right\}$. Then for all $i$,

$$
I^{(F+1)\left(C^{2}(n+D)+E\right)} S_{\mathfrak{Q}_{i}} \subseteq\left(\overline{(f) S_{\mathfrak{Q}_{i}}}\right)^{F+1} \subseteq(f) S_{\mathfrak{Q}_{i}} .
$$

Thus there exists an element $q$ of $S$ which does not lie in any $\mathfrak{Q}_{i}$ such that

$$
I^{(F+1)\left(C^{2}(n+D)+E\right)} S_{q} \subseteq(f) S_{q} .
$$

Now one proceeds as in the proof of [FHH, Theorem 1.4]. For completeness I will write it all out. 
Let $Y_{2}, \ldots, Y_{d}$ be indeterminates, $u_{i}=x_{1} Y_{i}-x_{i} \in R\left[Y_{2}, \ldots, Y_{d}\right]$. Let $p$ be the kernel of the epimorphism

$$
R\left[Y_{2}, \ldots, Y_{d}\right] \longrightarrow R\left[\frac{x_{2}}{x_{1}}, \ldots, \frac{x_{d}}{x_{1}}\right], Y_{i} \mapsto \frac{x_{i}}{x_{1}}
$$

Lift $q \in S$ to an element $q(Y) \in R\left[Y_{2}, \ldots, Y_{d}\right]$. Then we get that

$$
\left.I^{(F+1)\left(C^{2}(n+D)+E\right)} R\left[Y_{2}, \ldots, Y_{d}\right]_{q(Y)} \subseteq((f)+p)\right) R\left[Y_{2}, \ldots, Y_{d}\right]_{q(Y)} .
$$

By [FHH, Lemma 1.5] there exists an integer $G$ such that $I^{G} p \subseteq\left(u_{2}, \ldots, u_{d}\right)$. Hence

$$
I^{(F+1)\left(C^{2}(n+D)+E\right)+G} R\left[Y_{2}, \ldots, Y_{d}\right]_{q(Y)} \subseteq\left(\left(f, u_{2}, \ldots, u_{d}\right)\right) R\left[Y_{2}, \ldots, Y_{d}\right]_{q(Y)} .
$$

As $q(Y) \in R\left[Y_{2}, \ldots, Y_{d}\right] \backslash m R\left[Y_{2}, \ldots, Y_{d}\right]$ and since $R / m$ is infinite, we can specialize $Y_{2}, \ldots, Y_{d}$ to elements of $R$ such that the resulting $q(Y)$ is a unit of $R$. Then we immediately get

$$
I^{(F+1)\left(C^{2}(n+D)+E\right)+G} \subseteq\left(f, g_{2}, \ldots, g_{d}\right),
$$

where the $g_{i}$ are the specializations of the $u_{i}$ and hence the $g_{i}$ are elements of $I$. Now set $k=(F+1) C^{2}, l=(F+1)\left(C^{2} D+E\right)+G$.

A corollary is a weaker version of R. Hübl's main theorem:

Theorem A2 (cf. [FHH, Theorem 1.6]). Let $(R, \mathfrak{m})$ be a d-dimensional complete local domain containing the rationals, and let $I$ be an ideal in $R$. Then there exist positive integers $l$ and $k$ depending only on $I$ such that if $f \in \mathfrak{m}$ and $f \notin I^{n}$, then there exists a derivation $\delta \in \operatorname{Der}(R)$ such that $\delta(f) \notin I^{k n+l}$. Hence also $\delta(f) \notin I^{(k+l) n}$.

The proof follows exactly the lines of [FHH, Theorem 1.6], but using Theorem 1 from this note, rather than [FHH, Theorem 1.4].

Theorem A2 is weaker than R. Hübl's theorem because, for one thing, $k$ and $l$ from Theorem A1 depend on the ideal. In fact, in most rings there are no $k$ and $l$ which would make Theorem A1 work for all ideals:

Example. Let $R=F[[x, y]]$, where $F$ is a field and $x$ and $y$ are indeterminates over it. Suppose that there exist integers $k$ and $l$ such that for all $(x, y)$-primary ideals $I$, whenever $f \notin I^{n}$ then there exists $g \in I$ such that $I^{k n+l} \subseteq(f, g)$. In particular, let $I=\left(x^{k+l+1}, x y, y^{k+l+2}\right)$. Then $f=y^{k+l+1} \notin I$. By assumption, there exists an element $g=\alpha x^{k+l+1}+\beta x y+\gamma y^{k+l+2}$ such that $I^{k+l} \subseteq(f, g)$. In particular, $(x y)^{k+l}$ lies in $(f, g)$. Write

$$
(x y)^{k+l}=r y^{k+l+1}+s\left(\alpha x^{k+l+1}+\beta x y+\gamma y^{k+l+2}\right)
$$

for some $r, s \in R$. As $f, g$ form a regular sequence, necessarily $s=s_{0} y^{k+l}$ for some $s_{0}$ in $R$. Hence

$$
x^{k+l}=r y+s_{0}\left(\alpha x^{k+l+1}+\beta x y+\gamma y^{k+l+2}\right),
$$

which means that $x^{k+l} \in\left(x^{k+l+1}, y\right)$, a contradiction.

Now we return some more to Theorem A1. Note that in the case of a one-fibered ideal, in the proof of Theorem A1 we may take $C$ to be 1 and $F$ to be 0 so that $k=1$. It is open whether $k$ in Theorem A1 can always be taken to be 1 . 
Remark. The reason why the proof of Theorem A1 cannot allow $k=1$ in general is that it actually proves more. It clearly proves that

If $f \in \mathfrak{m}$ and $f \notin I^{n}$, then there exist $g_{2}, \ldots, g_{d} \in I$ such that $I^{k n+l} \subseteq$ $\left(f, g_{2}, \ldots, g_{d}\right)$ and for all Rees valuations $v$ of $I, v\left(I^{k n+l}\right) \geq v(f)$.

For ideals which are not one-fibered, it is too much to expect that $k$ with the additional assumption on the Rees valuations as above be 1 :

Proposition. Let I be an ideal in a d-dimensional Noetherian analytically unramified local ring $(R, \mathfrak{m})$ and $l$ a positive integer satisfying the following property:

If $f \in \mathfrak{m}$ and $f \notin I^{n}$, then there exist $g_{2}, \ldots, g_{d} \in I$ such that $I^{n+l} \subseteq$ $\left(f, g_{2}, \ldots, g_{d}\right)$ and for all Rees valuations $v$ of $I, v\left(I^{n+l}\right) \geq v(f)$.

Then I is one-fibered.

Proof. If $I$ is not one-fibered, then there exist two distinct Rees valuations $v_{1}$ and $v_{2}$ of $I$ and an element $f \in \mathfrak{m}$ such that $\frac{v_{1}(f)}{v_{1}(I)}<\frac{v_{2}(f)}{v_{2}(I)}$. Then for all integers $n \geq 1$, $f^{n v_{1}(I)}$ is not in $I^{n v_{1}(f)+1}$, as $v_{1}\left(f^{n v_{1}(I)}\right)=n v_{1}(I) v_{1}(f)<\left(n v_{1}(f)+1\right) v_{1}(I)=$ $v_{1}\left(I^{n v_{1}(f)+1}\right)$. By assumption then, for each $n \geq 1$, there exist $g_{2}, \ldots, g_{d}$ in $I$ such that $I^{n v_{1}(f)+1+l} \subseteq\left(f^{n v_{1}(I)}, g_{2}, \ldots, g_{d}\right)$ and $v_{2}\left(I^{n v_{1}(f)+1+l}\right) \geq v_{2}\left(f^{n v_{1}(I)}\right)$. Thus

$$
\left(n v_{1}(f)+1+l\right) v_{2}(I)=v_{2}\left(I^{n v_{1}(f)+1+l}\right) \geq v_{2}\left(f^{n v_{1}(I)}\right)=n v_{1}(I) v_{2}(f),
$$

and so for all $n \geq 1$,

$$
\frac{v_{1}(f)+\frac{1+l}{n}}{v_{1}(I)} \geq \frac{v_{2}(f)}{v_{2}(I)}
$$

Now $v_{1}(f), v_{1}(I), v_{2}(f), v_{2}(I)$ and $l$ are all independent of $n$, so in the limit as $n$ gets larger we get that $\frac{v_{1}(f)}{v_{1}(I)} \geq \frac{v_{2}(f)}{v_{2}(I)}$ which contradicts the assumption $\frac{v_{1}(f)}{v_{1}(I)}<\frac{v_{2}(f)}{v_{2}(I)}$.

In particular, consider the case when $R$ is a one-dimensional Noetherian analytically unramified local ring, $l$ is an integer and $I$ is an ideal satisfying the property that whenever $f \notin I^{n}$, then $I^{n+l} \subseteq(f)$. Then the proposition above implies that $I$ is one-fibered. This was already observed by Sally (see [FHH], page 323).

It is well known that in reasonable rings a fixed power of a given ideal lies in each of its reductions. In particular, if we choose $f$ as part of a minimal reduction of an $m$-primary ideal $I$, we can find $g_{2}, \ldots, g_{d}$ such that that fixed power of $I$ lies in $\left(f, g_{2}, \ldots, g_{d}\right)$. But Theorem A1 implies even more:

Corollary. Let $(R, \mathfrak{m})$ be a d-dimensional complete local domain with infinite residue field and let $I$ be an $\mathfrak{m}$-primary ideal. Then there exists an integer $l$ such that for any element $f \in I \backslash I^{2}$, there exist $g_{2}, \ldots, g_{d} \in I$ such that $I^{l}$ lies in $\left(f, g_{2}, \ldots, g_{d}\right)$.

\section{ACKNOWLEDGEMENT}

I thank William Heinzer for all the suggestions which helped improve the clarity of this presentation. 


\section{REFERENCES}

[BKKN] Berger, R., Kiehl, R., Kunz, E., Nastold, H.J.: Differentialrechnung in der analytischen Geometrie. Lecture Notes in Mathematics 38. 1967 MR 37:469

[F] Fedder, R.: A Frobenius characterization of rational singularity in two-dimensional graded rings. Trans. Amer. Math. Soc. 340, (1993), 655-668. MR 94b:13003

[FHH] Fedder, R., Huneke, C., Hübl, R.: Zeros of Differential Forms along One-Fibered Ideals. Proc. Amer. Math. Soc. 108, (1990), 319 - 325. MR 90d:13020

[Hn] C. Huneke: Uniform bounds in Noetherian rings, Invent. Math., 107 (1992), 203-223. MR 93b: 13027

[HS] Huneke, C., Smith, K.: Tight closure and the Kodaira Vanishing Theorem. J. reine angew. Math. 484 (1997), 127 - 152 MR 98e:13007

[KD] Kunz, E.: Kähler Differentials. Vieweg. Braunschweig, Wiesbaden, 1986. MR 88e:14025

[Li] Lipman J.: On Complete Ideals in Regular Local Rings. In: Algebraic Geometry and Commutative Algebra in Honor of Masayoshi NAGATA, (1988), 203-231. MR 90g:14003

[LiS] Lipman, J., Sathaye, A.: Jacobian Ideals and a Theorem of Briancon-Skoda. Michigan Math. J. 28 (1981), 199 -222. MR 83m:13001

[LiT] Lipman, J., Teissier, B.: Pseudo-Rational Local Rings and a Theorem of BrianconSkoda about Integral Closures of Ideals. Michigan Math. J. 28 (1981), 97-116. MR 82f: 14004

[R1] D. Rees: Izumi's Theorem, in "Commutative Algebra", editors M. Hochster, C. Huneke and J.D. Sally, Springer-Verlag, 1989, 407-416. MR 90g:13010

[R2] D. Rees: A note on analytically unramified local rings, J. London Math. Soc., 36 (1961), 24-28. MR 23:A3761

[SS1] Scheja, G., Storch, U.: Differentielle Eigenschaften der Lokalisierungen analytischer Algebren. Math. Annalen 197, (1972), 137-170. MR 46:5299

[SS2] Scheja, G., Storch, U.: Über differentielle Abhängigkeit bei Idealen analytischer Algebren. Math. Z. 114 (1970), 101-112. MR 41:8408

[SW] Scheja, G., Wiebe, H.: Über Derivationen von lokalen analytischen Algebren. Symp. Math. XI (1973), 161-192. MR 49:3225

NWF I - Mathematik, Universität Regensburg, 93040 Regensburg, Germany

E-mail address: Reinhold.Huebl@Mathematik.Uni-Regensburg.de

Department of Mathematical Sciences, New Mexico State University, Las Cruces, New MeXico 88003

E-mail address: iswanson@mnsu.edu 